\section{A PRELIMINARY OBSERVATION ON THE BIRDS OF LONAR CRATER LAKE, BULDHANA DISTRICT, MAHARASHTRA}

\section{Muhamed Jafer Palot}

Western Ghats Field Research Station, Zoological Survey of India, Kozhikode, Kerala 673002, India

Email: madayipara@rediffmail.com

Lonar lake $\left(19^{\circ} 59^{\prime} \mathrm{N}-74^{\circ} 34^{\prime} \mathrm{E}\right)$ is situated in Mehkar taluka of Buldhana district of Maharashtra. This is the only crater in basaltic rock formed by the meteoritic impact in India, ranking third largest in the world. The Lonar crater has a circular outline with a diameter of $1830 \mathrm{~m}$ and a depth of $150 \mathrm{~m}$ with steep vertical slopes. The temperature fluctuates between $27^{\circ}-45^{\circ} \mathrm{C}$ from winter to summer and rainfall from 500 to $800 \mathrm{~mm}$.

Voluminous information has been generated on the geochemistry and water quality parameters of this unique lake system (Badve et al., 1993). Very few works have been done on the flora and fauna of this habitat. A report prepared by the Econet, Pune (Anonymous, 1999), listed 36 species of birds from the region. The nomenclature and classification is after Manakadan \& Pittie (2001).

While conducting hydrobiological studies on the Lonar Lake from 1-7 November 2000, we observed and maintained a record of the birds of the area from different habitats, including inhabited open areas, scrub jungles of the slopes, the saline lake and its basins. Birds observed in these habitats were segregated into Common (C), Uncommon (UC), Rare (R) and Not Found (NF) categories based on their occurrence during the survey. The characteristics of the three important habitats are as follows.

(i) Crater basin and lake: The lake is mostly circular in shape except on its north-eastern side, where siltation caused by the Dhara (a perennial stream) has created small mudflats. The shallow nature of the habitat has provided good growth of aquatic reeds and sedges dominated by Typha angustifolia, Fimbristylis sp., Cyperus sp., etc. The most striking feature of the lake is its extreme salinity and high alkalinity $(\mathrm{pH}$ above 10.5) due to the confined nature of the lake, bounded by the crater wall. Average depth of the lake varies from $2 \mathrm{~m}$ in summer to $4 \mathrm{~m}$ in rainy season. The flat lake basin area is covered with natural forests in some parts, while some parts are planted with Eucalyptus sp., Delonyx regia, Polyalthia longifolia, Leucaena leucocephala, Prosopis juliflora, etc. by the afforestation programme of the forest department. There is a 21 ha patch of cultivated land of banana and other vegetables at the base of the ravine fed by the perennial streams.

(ii) Slopes of the crater: The slopes of the crater are quite steep and descend to an average depth of $136 \mathrm{~m}$ as measured from the crest of the rim. The eastern slopes are gentler with a broad gully formation in the north-eastern part towards Lonar town, where a perennial spring originates and flows into the crater. The two main streams are Dhara and Sita-Nahani. The slopes are covered with bushy vegetation over most of the parts. The vegetation appears to be of a stunted form and completely deciduous in nature, dominated with Butea monosperma, Aegle marmelos, Zizyphus oenoplia, Lantana camara, Cassia auriculata, Capparis sp. etc.

(iii) The rim and outside crater: The Lonar crater has a $30 \mathrm{~m}$ high rim with a diameter averaging $1830 \mathrm{~m}$ at the rim crest; the northeastern side is much open. The outside of the crater has very gentle slopes, which eventually merges with the surrounding countryside interspersed with agricultural lands.

Even though the Lonar crater has a very small area, the diversity of different micro-ecosystems and the micro-climatic conditions accommodates a total of 110 species of birds belonging to 41 families (Table 1). Of these, 22 are migratory, 80 are residents and eight are considered to be local migrants (Abdulali, 1981). The number of species recorded was high on the rim and outside the crater (91), followed by slopes of the crater (89) and 35 species from the lake and its basin. Birds such as Small Blue Kingfisher, White-breasted Kingfisher, Small Bee-eater, Common Swallow, Wire-tailed Swallow, Redrumped Swallow, Indian Great Reed Warbler, Blyth's Reed Warbler, Paddyfield Pipit, Richard's Pipit, Grey Wagtail and Large Pied Wagtail were generalists present in all the three habitats. A good number of Indian Peafowl were observed mainly on the slopes of the crater.

Migratory waterfowls such as Brahminy Shelduck, Northern Shoveller, Marsh Sandpiper, Green Sandpiper, Wood Sandpiper and Common Sandpiper were found to be using the lake basin area for foraging. Even though the lake water is without fish species, some piscivorous species like little cormorants, egrets and herons inhabit the lake water area. Even though the lake water is highly saline, freshwater bird species such as Green Sandpiper and duck species were found to use the lake as a foraging ground. Presence of these species is due to the inflow of the perennial freshwater streams at the northeastern area of the lake.

A roosting population of waterfowls including herons, egrets and ibises were observed on the north-eastern lake basin area. The bushy growth of Prosopis juliflora and Acacia sp. was observed to accommodate as much as 1000 s of waterfowls to the area. The roosting birds were observed to arrive by $17.5 \mathrm{ohr}$ and occupy the roost by $18.40 \mathrm{hr}$. Most of them were seen coming from the eastern and north-eastern direction. Their return flight started from early morning by $05.5 \mathrm{ohr}$ and lasted up to $07.15 \mathrm{hrs}$. The roost was dominated with Median Egrets followed by Little Egrets, Pond Herons, Cattle Egrets, Large Egrets and White Ibis.

\section{REFERENCES}

Abdulali, H. (1981). Checklist of The Birds of Maharashtra with Notes on their Status Around Bombay. Bombay Natural History Society, Mumbai. Anonymous (1999). Rapid environmental assessment and the conservation and management plan for Lonar crater, Buldhana. Final Report. Prepared by Econet, Pune.

Badave, R.M., K.P.N. Kumara \& C. Rajashekhar (1993). Eutrophication of Lonar Lake, Maharashtra. Current Science 65(4): 347-351.

Manakadan, R. \& A. Pittie (2001). Standardized Common and Scientific names of the Birds of the Indian Subcontinent. BUCEROS. Envis Nerwsletter: Avian Ecology \& Inland Wetlands. Vol.6, No.1. Bombay Natural History Society, Mumbai.

Manuscript 1125; (C) ZOO; Date of publication 21 December 2006 Received 11 December 2003; Revised received 30 August 2006; Finally accepted 08 December 2006 
Table 1. Systematic list of birds observed at Lonar crater lake, Maharashtra

\begin{tabular}{|c|c|c|c|c|c|c|}
\hline \multirow{2}{*}{\multicolumn{2}{|c|}{ Scientific Name }} & \multirow[t]{2}{*}{ Common Name } & \multirow[t]{2}{*}{ Status } & \multicolumn{3}{|c|}{ Occurrence } \\
\hline & & & & Lake & Rim & Outside \\
\hline 1 & $\begin{array}{l}\text { Family: Podicipididae } \\
\text { Tachybaptus ruficollis (Pallas) }\end{array}$ & Little Grebe & $\mathrm{R}$ & C & $\mathrm{NF}$ & C \\
\hline 2 & $\begin{array}{l}\text { Family: Phalacrocoracidae } \\
\text { Phalacrocorax niger(Vieillot) }\end{array}$ & Little Cormorant & $\mathrm{R}$ & $\mathrm{R}$ & $\mathrm{NF}$ & C \\
\hline $\begin{array}{l}3 \\
4 \\
5 \\
6 \\
7\end{array}$ & $\begin{array}{l}\text { Family: Ardeidae } \\
\text { Ardea cinerea Linnaeus } \\
\text { Ardeola grayii (Sykes) } \\
\text { Bubulcus ibis (Linnaeus) } \\
\text { Mesophoyx intermedia (Wagler) } \\
\text { Egretta garzetta (Linnaeus) }\end{array}$ & $\begin{array}{l}\text { Grey Heron } \\
\text { Indian Pond Heron } \\
\text { Cattle Egret } \\
\text { Median Egret } \\
\text { Little Egret }\end{array}$ & $\begin{array}{l}\text { LM } \\
R \\
R \\
R \\
R\end{array}$ & $\begin{array}{l}\text { R } \\
\text { UC } \\
C \\
C \\
\text { UC }\end{array}$ & $\begin{array}{l}\text { NF } \\
\text { UC } \\
\text { C } \\
\text { NF } \\
\text { NF }\end{array}$ & $\begin{array}{l}\text { UC } \\
\mathrm{C} \\
\mathrm{C} \\
\mathrm{C} \\
\mathrm{C}\end{array}$ \\
\hline 8 & $\begin{array}{l}\text { Family: Threskiornithidae } \\
\text { Threskiornis melanocephalus (Latham) }\end{array}$ & Oriental White Ibis & LM & $\mathrm{R}$ & $\mathrm{NF}$ & NF \\
\hline $\begin{array}{l}9 \\
10 \\
11\end{array}$ & $\begin{array}{l}\text { Family: Anatidae } \\
\text { Tadorna ferruginea (Pallas) } \\
\text { Anas poecilorhyncha J.R. Forester } \\
\text { Anas clypeata Linnaeus }\end{array}$ & $\begin{array}{l}\text { Brahminy Shelduck } \\
\text { Spot-billed Duck } \\
\text { Northern Shoveller }\end{array}$ & $\begin{array}{l}M \\
R \\
M\end{array}$ & $\begin{array}{l}\mathrm{R} \\
\mathrm{R} \\
\mathrm{C}\end{array}$ & $\begin{array}{l}\text { NF } \\
\text { NF } \\
\text { NF }\end{array}$ & $\begin{array}{l}\text { NF } \\
\text { UC } \\
\text { C }\end{array}$ \\
\hline $\begin{array}{l}12 \\
13\end{array}$ & $\begin{array}{l}\text { Family: Accipitridae } \\
\text { Elanus caeruleus (Desfontaines) } \\
\text { Accipiter badius (Gmelin) }\end{array}$ & $\begin{array}{l}\text { Black-shouldered Kite } \\
\text { Shikra }\end{array}$ & $\begin{array}{l}\mathrm{LM} \\
\mathrm{R}\end{array}$ & $\begin{array}{l}\text { NF } \\
\text { NF }\end{array}$ & $\begin{array}{l}\text { UC } \\
\text { UC }\end{array}$ & $\begin{array}{l}\text { UC } \\
\text { UC }\end{array}$ \\
\hline 14 & $\begin{array}{l}\text { Family: Falconidae } \\
\text { Falco tinnunculus Linnaeus }\end{array}$ & Common Kestrel & M & $\mathrm{NF}$ & UC & NF \\
\hline $\begin{array}{l}15 \\
16\end{array}$ & $\begin{array}{l}\text { Family: Phasianidae } \\
\text { Perdicula argoondah (Sykes) } \\
\text { Pavo cristatus Linnaeus }\end{array}$ & $\begin{array}{l}\text { Rock Bush Quail } \\
\text { Indian Peafowl }\end{array}$ & $\begin{array}{l}\mathrm{R} \\
\mathrm{R}\end{array}$ & $\begin{array}{l}\text { NF } \\
\text { NF }\end{array}$ & $\begin{array}{l}\text { UC } \\
\text { C }\end{array}$ & $\begin{array}{l}\mathrm{R} \\
\mathrm{R}\end{array}$ \\
\hline $\begin{array}{l}17 \\
18 \\
19 \\
20\end{array}$ & $\begin{array}{l}\text { Family: Rallidae } \\
\text { Amauorornis phoenicurus (Pennant) } \\
\text { Gallinula chloropus (Linnaeus) } \\
\text { Porphyrio porphyrio (Linnaeus) } \\
\text { Fulica atra Linnaeus }\end{array}$ & $\begin{array}{l}\text { White-breasted Waterhen } \\
\text { Common Moorhen } \\
\text { Purple Moorhen } \\
\text { Common Coot }\end{array}$ & $\begin{array}{l}R \\
R \\
R \\
M\end{array}$ & $\begin{array}{l}\mathrm{C} \\
\mathrm{R} \\
\mathrm{UC} \\
\mathrm{UC}\end{array}$ & $\begin{array}{l}\text { R } \\
\text { NF } \\
\text { NF } \\
\text { NF }\end{array}$ & $\begin{array}{l}\mathrm{C} \\
\mathrm{NF} \\
\mathrm{R} \\
\mathrm{UC}\end{array}$ \\
\hline 21 & $\begin{array}{l}\text { Family: Recurvirostridae } \\
\text { Himantopus himantopus (Linnaeus) }\end{array}$ & Black-winged Stilt & $\mathrm{R}$ & $\mathrm{C}$ & $\mathrm{NF}$ & $\mathrm{C}$ \\
\hline 22 & $\begin{array}{l}\text { Family: Charadriidae } \\
\text { Vanellus indicus (Boddaert) }\end{array}$ & Red-wattled Lapwing & $\mathrm{R}$ & C & UC & C \\
\hline $\begin{array}{l}23 \\
24 \\
25 \\
26\end{array}$ & $\begin{array}{l}\text { Family: Scolopacidae } \\
\text { Tringa stagnatilis (Linnaeus) } \\
\text { Tringa ochropus Linnaeus } \\
\text { Tringa glareola Linnaeus } \\
\text { Actitis hypoleucos Linnaeus }\end{array}$ & $\begin{array}{l}\text { Marsh Sandpiper } \\
\text { Green Sandpiper } \\
\text { Wood Sandpiper } \\
\text { Common Sandpiper }\end{array}$ & $\begin{array}{l}M \\
M \\
M \\
M\end{array}$ & $\begin{array}{l}\mathrm{C} \\
\mathrm{C} \\
\mathrm{UC} \\
\mathrm{C}\end{array}$ & $\begin{array}{l}\text { R } \\
\text { UC } \\
\text { NF } \\
\text { C }\end{array}$ & $\begin{array}{l}\text { UC } \\
\text { C } \\
\text { UC } \\
C\end{array}$ \\
\hline 27 & $\begin{array}{l}\text { Family: Laridae } \\
\text { Sterna aurantia J.E. Gray }\end{array}$ & River Tern & LM & $\mathrm{NF}$ & $\mathrm{NF}$ & UC \\
\hline $\begin{array}{l}28 \\
29 \\
30 \\
31 \\
32\end{array}$ & $\begin{array}{l}\text { Family: Columbidae } \\
\text { Streptopelia orientalis (Latham) } \\
\text { Streptopelia decaocto (Frivaldszky) } \\
\text { Streptopelia senegalensis (Linnaeus) } \\
\text { Streptopelia chinensis (Scopoli) } \\
\text { Chalcophaps indica (Linnaeus) }\end{array}$ & $\begin{array}{l}\text { Oriental Turtle Dove } \\
\text { Eurasian Collared Dove } \\
\text { Little Brown Dove } \\
\text { Spotted Dove } \\
\text { Emerald Dove }\end{array}$ & $\begin{array}{l}R \\
L M \\
R \\
R \\
R\end{array}$ & $\begin{array}{l}\text { NF } \\
\text { NF } \\
\text { NF } \\
\text { NF } \\
\text { NF }\end{array}$ & $\begin{array}{l}\text { UC } \\
\text { UC } \\
\text { C } \\
\text { NF } \\
\text { R }\end{array}$ & $\begin{array}{l}\text { UC } \\
\text { C } \\
\text { C } \\
\text { UC } \\
\text { NF }\end{array}$ \\
\hline 33 & $\begin{array}{l}\text { Family: Psittacidae } \\
\text { Psittacula krameri (Scopoli) }\end{array}$ & Rose-ringed Parakeet & $\mathrm{R}$ & $\mathrm{NF}$ & C & C \\
\hline $\begin{array}{l}34 \\
35 \\
36 \\
37\end{array}$ & $\begin{array}{l}\text { Family: Cuculidae } \\
\text { Clamator jacobinus (Boddaert) } \\
\text { Hierococcyx varius (Vahl) } \\
\text { Eudynamys scolopacea (Linnaeus) } \\
\text { Centropus sinensis (Stephens) }\end{array}$ & $\begin{array}{l}\text { Pied Crested Cuckoo } \\
\text { Brainfever Bird } \\
\text { Asian Koel } \\
\text { Greater Coucal }\end{array}$ & $\begin{array}{l}\mathrm{BM} \\
\mathrm{R} \\
\mathrm{R} \\
\mathrm{R}\end{array}$ & $\begin{array}{l}\text { NF } \\
\text { NF } \\
\text { NF } \\
\text { NF }\end{array}$ & $\begin{array}{l}\mathrm{NF} \\
\mathrm{R} \\
\mathrm{C} \\
\mathrm{C}\end{array}$ & $\begin{array}{l}\text { UC } \\
R \\
C \\
C\end{array}$ \\
\hline 38 & $\begin{array}{l}\text { Family: Tytonidae } \\
\text { Tyto alba (Scopoli) }\end{array}$ & Barn Owl & $\mathrm{R}$ & $\mathrm{NF}$ & $\mathrm{R}$ & $\mathrm{R}$ \\
\hline $\begin{array}{l}39 \\
40\end{array}$ & $\begin{array}{l}\text { Family: Strigidae } \\
\text { Bubo bubo (Linnaeus) } \\
\text { Athene brama (Temminck) }\end{array}$ & $\begin{array}{l}\text { Eurasian Eagle Owl } \\
\text { Spotted Owlet }\end{array}$ & $\begin{array}{l}\mathrm{R} \\
\mathrm{R}\end{array}$ & $\begin{array}{l}\text { NF } \\
\text { NF }\end{array}$ & $\begin{array}{l}\mathrm{R} \\
\mathrm{C}\end{array}$ & $\begin{array}{l}\mathrm{R} \\
\mathrm{C}\end{array}$ \\
\hline 41 & $\begin{array}{l}\text { Family: Caprimulgidae } \\
\text { Caprimulgus spp }\end{array}$ & Nightjar? & $\mathrm{R}$ & NF & UC & UC \\
\hline 42 & $\begin{array}{l}\text { Family: Apodidae } \\
\text { Apus affinis (J.E. Gray) }\end{array}$ & House Swift & $\mathrm{R}$ & NF & UC & $\mathrm{C}$ \\
\hline
\end{tabular}




\begin{tabular}{|c|c|c|c|c|c|c|}
\hline & \multirow[t]{2}{*}{ Scientific Name } & \multirow[t]{2}{*}{ Common Name } & \multirow[t]{2}{*}{ Status } & \multicolumn{3}{|c|}{ Occurrence } \\
\hline & & & & Lake & Rim & Outside \\
\hline & Family: Alcedinidae & & & & & \\
\hline 43 & Alcedo atthis (Linnaeus) & Small Blue Kingfisher & $\mathrm{R}$ & $\mathrm{R}$ & UC & C \\
\hline 44 & Halcyon smyrnensis (Linnaeus) & White-breasted Kingfisher & $\mathrm{R}$ & C & UC & C \\
\hline 45 & $\begin{array}{l}\text { Family: Meropidae } \\
\text { Merops orientalis Latham }\end{array}$ & Small Bee-eater & $\mathrm{R}$ & C & C & C \\
\hline 46 & $\begin{array}{l}\text { Family: Coraciidae } \\
\text { Coracias benghalensis (Linnaeus) }\end{array}$ & Indian Roller & $\mathrm{R}$ & NF & $\mathrm{R}$ & C \\
\hline 47 & $\begin{array}{l}\text { Family: Upupidae } \\
\text { Upupa epops Linnaeus }\end{array}$ & Common Hoopoe & $\mathrm{R}$ & NF & UC & $\mathrm{C}$ \\
\hline 48 & $\begin{array}{l}\text { Family: Bucerotidae } \\
\text { Ocyceros birostris (Scopoli) }\end{array}$ & Indian Grey Hornbill & $\mathrm{R}$ & NF & UC & UC \\
\hline $\begin{array}{l}49 \\
50 \\
51\end{array}$ & $\begin{array}{l}\text { Family: Capitonidae } \\
\text { Megalaima zeylanica (Gmelin) } \\
\text { Megalaima rubrticapilla (Gmelin) } \\
\text { Megalaima haemacephala (Muller) }\end{array}$ & $\begin{array}{l}\text { Brown- headed Barbet } \\
\text { Crimson-throated Barbet } \\
\text { Coppersmith Barbet }\end{array}$ & $\begin{array}{l}R \\
R \\
R\end{array}$ & $\begin{array}{l}\text { NF } \\
\text { NF } \\
\text { NF }\end{array}$ & $\begin{array}{l}\mathrm{NF} \\
\mathrm{R} \\
\mathrm{C}\end{array}$ & $\begin{array}{l}\text { R } \\
N F \\
\text { UC }\end{array}$ \\
\hline $\begin{array}{l}52 \\
53 \\
54\end{array}$ & $\begin{array}{l}\text { Family: Alaudidae } \\
\text { Eremopterix grisea (Scopoli) } \\
\text { Ammomanes phoenicurus (Franklin) } \\
\text { Calandrella brachydactyla (Leisler) }\end{array}$ & $\begin{array}{l}\text { Ashy Crowned Finch Lark } \\
\text { Rufous-tailed Finch Lark } \\
\text { Greater Short- toed Lark }\end{array}$ & $\begin{array}{l}R \\
R \\
M\end{array}$ & $\begin{array}{l}\text { NF } \\
\text { NF } \\
\text { NF }\end{array}$ & $\begin{array}{l}\text { UC } \\
\text { R } \\
\text { NF }\end{array}$ & $\begin{array}{l}\mathrm{C} \\
\mathrm{UC} \\
\mathrm{C}\end{array}$ \\
\hline $\begin{array}{l}55 \\
56 \\
57 \\
58\end{array}$ & $\begin{array}{l}\text { Family: Hirundinidae } \\
\text { Hirundo concolor Sykes } \\
\text { Hirundo rustica Linnaeus } \\
\text { Hirundo smithii Leach } \\
\text { Hirundo daurica Linnaeus }\end{array}$ & $\begin{array}{l}\text { Dusky Crag Martin } \\
\text { Common Swallow } \\
\text { Wire-tailed Swallow } \\
\text { Red-rumped Swallow }\end{array}$ & $\begin{array}{l}\mathrm{R} \\
\mathrm{M} \\
\mathrm{R} \\
\mathrm{R}\end{array}$ & $\begin{array}{l}\text { NF } \\
\mathrm{C} \\
\mathrm{C} \\
\mathrm{UC}\end{array}$ & $\begin{array}{l}\text { UC } \\
C \\
\text { R } \\
\text { UC }\end{array}$ & $\begin{array}{l}\text { UC } \\
C \\
\text { R } \\
\text { UC }\end{array}$ \\
\hline $\begin{array}{l}59 \\
60\end{array}$ & $\begin{array}{l}\text { Family: Laniidae } \\
\text { Lanius excubitor Linnaeus } \\
\text { Lanius vittatus Valenciennes }\end{array}$ & $\begin{array}{l}\text { Great Grey Shrike } \\
\text { Bay-backed Shrike }\end{array}$ & $\begin{array}{l}\mathrm{R} \\
\mathrm{R}\end{array}$ & $\begin{array}{l}\text { NF } \\
\text { NF }\end{array}$ & $\begin{array}{l}\mathrm{R} \\
\mathrm{UC}\end{array}$ & $\begin{array}{l}\text { UC } \\
\mathrm{C}\end{array}$ \\
\hline 61 & $\begin{array}{l}\text { Family: Oriolidae } \\
\text { Oriolus oriolus (Linnaeus) }\end{array}$ & Eurasian Golden Oriole & LM & NF & $\mathrm{R}$ & NF \\
\hline $\begin{array}{l}62 \\
63 \\
64\end{array}$ & $\begin{array}{l}\text { Family: Dicruruidae } \\
\text { Dicrurus macrocercus Vieillot } \\
\text { Dicrurus leucophaeus Vieillot } \\
\text { Dicrurus caerulescens (Linnaeus) }\end{array}$ & $\begin{array}{l}\text { Black Drongo } \\
\text { Ashy Drongo } \\
\text { White-bellied Drongo }\end{array}$ & $\begin{array}{l}\mathrm{R} \\
\mathrm{M} \\
\mathrm{R}\end{array}$ & $\begin{array}{l}\text { NF } \\
\text { NF } \\
\text { NF }\end{array}$ & $\begin{array}{l}\text { UC } \\
\text { R } \\
\text { UC }\end{array}$ & $\begin{array}{l}\mathrm{C} \\
\mathrm{NF} \\
\mathrm{NF}\end{array}$ \\
\hline $\begin{array}{l}65 \\
66\end{array}$ & $\begin{array}{l}\text { Family: Sturnidae } \\
\text { Sturnus pagodarum (Gmelin) } \\
\text { Acridotheres tristis (Linnaeus) }\end{array}$ & $\begin{array}{l}\text { Brahminy Myna } \\
\text { Common Myna }\end{array}$ & $\begin{array}{l}\mathrm{R} \\
\mathrm{R}\end{array}$ & $\begin{array}{l}\text { NF } \\
\text { NF }\end{array}$ & $\begin{array}{l}\mathrm{C} \\
\mathrm{C}\end{array}$ & $\begin{array}{l}\mathrm{C} \\
\mathrm{C}\end{array}$ \\
\hline $\begin{array}{l}67 \\
68 \\
69\end{array}$ & $\begin{array}{l}\text { Family: Corvidaae } \\
\text { Corvus splendens Vieillot } \\
\text { Corvus macrorhynchos Wagler } \\
\text { Dendrocitta vagabunda (Latham) }\end{array}$ & $\begin{array}{l}\text { House Crow } \\
\text { Jungle Crow } \\
\text { Indian Treepie }\end{array}$ & $\begin{array}{l}R \\
R \\
R\end{array}$ & $\begin{array}{l}\text { NF } \\
\text { NF } \\
\text { NF }\end{array}$ & $\begin{array}{l}\mathrm{C} \\
\mathrm{C} \\
\mathrm{C}\end{array}$ & $\begin{array}{l}\mathrm{C} \\
\mathrm{C} \\
\mathrm{C}\end{array}$ \\
\hline $\begin{array}{l}70 \\
71\end{array}$ & $\begin{array}{l}\text { Family; Campephagidae } \\
\text { Coracina macei (Lesson) } \\
\text { Tephrodornis pondicerianus (Gmelin) }\end{array}$ & $\begin{array}{l}\text { Large Cuckoo Shrike } \\
\text { Common Wood Shrike }\end{array}$ & $\begin{array}{l}\mathrm{R} \\
\mathrm{R}\end{array}$ & $\begin{array}{l}\text { NF } \\
\text { NF }\end{array}$ & $\begin{array}{l}\mathrm{R} \\
\mathrm{R}\end{array}$ & $\begin{array}{l}\text { NF } \\
\text { UC }\end{array}$ \\
\hline 72 & $\begin{array}{l}\text { Family: Irenidae } \\
\text { Aegithina tiphia (Linnaeus) }\end{array}$ & Common lora & $\mathrm{R}$ & NF & C & C \\
\hline 73 & $\begin{array}{l}\text { Family: Pycnonotidae } \\
\text { Pycnonotus cafer (Linnaeus) }\end{array}$ & Red-vented Bulbul & $\mathrm{R}$ & $\mathrm{NF}$ & $\mathrm{C}$ & C \\
\hline & $\begin{array}{l}\text { Family: Muscicapidae } \\
\text { Subfamily: Timalinae }\end{array}$ & & & & & \\
\hline 74 & Chrysomma sinense (Gmelin) & Yellow-eyed Babbler & $\mathrm{R}$ & NF & $\mathrm{R}$ & NF \\
\hline 75 & Turdoides caudatus (Dumont) & Common Babbler & $\mathrm{R}$ & NF & $\mathrm{R}$ & NF \\
\hline 76 & Turdoides malcolmi(Sykes) & Large Grey Babbler & $\mathrm{R}$ & NF & $\mathrm{C}$ & C \\
\hline 77 & Turdoides striatus (Dumont) & Jungle Babbler & $\mathrm{R}$ & NF & $\mathrm{C}$ & UC \\
\hline 78 & $\begin{array}{l}\text { Subfamily: Muscicapinae } \\
\text { Muscicapa daurica Pallas }\end{array}$ & Asian Brown Flycatcher & LM & $\mathrm{NF}$ & $\mathrm{R}$ & NF \\
\hline 79 & $\begin{array}{l}\text { Subfamily: Monarchinae } \\
\text { Terpsiphone paradisi (Linnaeus) }\end{array}$ & Paradise Flycatcher & $\mathrm{R}$ & NF & UC & NF \\
\hline 80 & $\begin{array}{l}\text { Subfamily: Rhipidurinae } \\
\text { Rhipidura albicollis (Vieillot) }\end{array}$ & White-throated Fantail Flycatcher & M & NF & UC & NF \\
\hline 81 & $\begin{array}{l}\text { Subfamily: Sylvinae } \\
\text { Cisticola juncidis (Rafinseque) }\end{array}$ & Streaked Fantail Warbler & $\mathrm{R}$ & NF & $\mathrm{R}$ & UC \\
\hline 82 & Prinia inornata Sykes & Plain Prinia & $\mathrm{R}$ & NF & UC & $\mathrm{R}$ \\
\hline 83 & Prinia socialis Sykes & Ashy Prinia & $\mathrm{R}$ & NF & $\mathrm{C}$ & UC \\
\hline
\end{tabular}




\begin{tabular}{|c|c|c|c|c|c|c|}
\hline & Scientific Name & Common Name & Status & & curre & \\
\hline & & & & Lake & Rim & Outside \\
\hline 84 & Orthotomus sutorius (Pennant) & Common Tailor Bird & $\mathrm{R}$ & $\mathrm{NF}$ & C & $\mathrm{C}$ \\
\hline 85 & Acrocephalus stentoreus (Emprich \& Ehrenberg) & Indian Great Reed Warbler & $\mathrm{R}$ & C & C & $\mathrm{C}$ \\
\hline 86 & Acrocephalus dumetorum Blyth & Blyth's Reed Warbler & M & $\mathrm{C}$ & $\mathrm{C}$ & UC \\
\hline 87 & Sylvia curruca (Linnaeus) & Common Lesser Whitethroat & M & NF & UC & UC \\
\hline 88 & Phylloscopus trochiloides (Sundevall) & Greenish Leaf Warbler & M & $\mathrm{NF}$ & $\mathrm{C}$ & $\mathrm{C}$ \\
\hline 89 & Phylloscopus occipitalis (Blyth) & Western Crowned Warbler & M & $\mathrm{NF}$ & $\mathrm{R}$ & $\mathrm{R}$ \\
\hline & Subfamily: Turdinae & & & & & \\
\hline 90 & Copsychus saularis (Linnaeus) & Oriental Magpie Robin & $\mathrm{R}$ & NF & C & C \\
\hline 91 & Cercomela fusca (Blyth) & Indian Chat & $\mathrm{R}$ & NF & UC & UC \\
\hline 92 & Saxicola carprata (Linnaeus) & Pied Bush Chat & $\mathrm{R}$ & NF & C & UC \\
\hline 93 & Saxicoloides fulicata (Linnaeus) & Indian Robin & $\mathrm{R}$ & NF & $\mathrm{C}$ & C \\
\hline 94 & Monticola solitarius (Linnaeus) & Blue Rock Thrush & M & NF & UC & NF \\
\hline 95 & $\begin{array}{l}\text { Family: Paridae } \\
\text { Parus major Linnaeus }\end{array}$ & Great Tit & $\mathrm{R}$ & NF & UC & NF \\
\hline & Family: Motacillidae & & & & & \\
\hline 96 & Anthus trivialis (Linnaeus) & Eurasian Tree Pipit & M & NF & UC & UC \\
\hline 97 & Anthus rufulus Vieillot & Paddyfield Pipit & $\mathrm{R}$ & C & C & $\mathrm{C}$ \\
\hline 98 & Anthus richardi Vieillot & Richard's Pipit & M & $\mathrm{R}$ & UC & $\mathrm{R}$ \\
\hline 99 & Motacilla citreola Pallas & Citrine Wagtail & M & UC & $\mathrm{R}$ & NF \\
\hline 100 & Motacilla cinerea Tunstall & Grey Wagtail & M & C & C & C \\
\hline 101 & Motacilla alba Linnaeus & White Wagtail & M & UC & $\mathrm{NF}$ & NF \\
\hline 102 & Motacilla maderaspatensis Gmelin & Large Pied Wagtail & $\mathrm{R}$ & UC & $\mathrm{R}$ & C \\
\hline 103 & $\begin{array}{l}\text { Family: Dicaeidae } \\
\text { Dicaeum agile Tickell }\end{array}$ & Thick-billed Flowerpecker & $\mathrm{R}$ & $\mathrm{NF}$ & UC & UC \\
\hline & Family: Nectariniidae & & & & & \\
\hline 104 & Nectarinia asiatica (Latham) & Purple Sunbird & $\mathrm{R}$ & NF & C & $\mathrm{C}$ \\
\hline 105 & $\begin{array}{l}\text { Family: Zosteropidae } \\
\text { Zosterops palpebrosa (Temminck) }\end{array}$ & Oriental White-eye & $\mathrm{R}$ & NF & $\mathrm{R}$ & $\mathrm{R}$ \\
\hline & $\begin{array}{l}\text { Family: Passeridae } \\
\text { Subamily: Passerinae }\end{array}$ & & & & & \\
\hline 106 & Passer domesticus (Linnaeus) & House Sparrow & $\mathrm{R}$ & NF & UC & C \\
\hline 107 & Petronia xanthocollis (Burton) & Yellow-throated Sparrow & $\mathrm{R}$ & NF & UC & UC \\
\hline 108 & $\begin{array}{l}\text { Subfamily: Ploceinae } \\
\text { Ploceus philippinus (Linnaeus) }\end{array}$ & Baya Weaver & $\mathrm{R}$ & NF & C & UC \\
\hline & Family: Estrildae & & & & & \\
\hline 109 & Lonchura malabarica (Linnaeus) & White-throated Munia & $\mathrm{R}$ & NF & C & UC \\
\hline 110 & Lonchura punctulata (Linnaeus) & Spotted Munia & $\mathrm{R}$ & NF & NF & UC \\
\hline
\end{tabular}

Status: R - Resident; M - Migrant; LM - Local Migrant; BM - Breeding Migrant Occurrence: C - Common; UC - Uncommon; R - Rare; NF - Not Found

\section{ACKNOWLedgements}

The author is grateful to the Director, Zoological Survey of India, Kolkata and Officer-in-Charge, Freshwater Biological Station, Zoological Survey of India, Hyderabad for facilities and encouragements. I am grateful to the Chief Wildlife Warden, Maharashtra Forest Department and his subordinates at Buldhana district for permission and cooperation. My sincere thanks are also due to Shri. C. Radhakrishnan, Officer-inCharge, Zoological Survey of India, Kozhikode for encouragement, for going through the manuscript and making valuable comments.

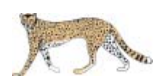

